\title{
MPP1-based mechanism of resting state raft organization in the plasma membrane. Is it a general or specialized mechanism in erythroid cells?
}

\author{
Magdalena Trybus, Lukasz Niemiec, Agnieszka Biernatowska, \\ Anita Hryniewicz-Jankowska, Aleksander F. Sikorski
}

Department of Cytobiochemistry, Faculty of Biotechnology, University of Wroclaw

\begin{abstract}
Biological membranes are organized in various microdomains, one of the best known being called membrane rafts. The major function of these is thought to organize signaling partners into functional complexes. An important protein found in membrane raft microdomains of erythroid and other blood cells is MPP1 (membrane palmitoylated protein 1)/p55. MPP1 (p55) belongs to the MAGUK (membrane-associated guanylate kinase homolog) family and it is a major target of palmitoylation in the red blood cells (RBCs) membrane. The wellknown function of this protein is to participate in formation of the junctional complex of the erythrocyte membrane skeleton. However, its function as a "raft organizer" is not well understood. In this review we focus on recent reports concerning MPP1 participation in membrane rafts organization in erythroid cells, including its role in signal transduction. Currently it is not known whether MPP1 could have a similar role in cell types other than erythroid lineage. We present also preliminary data regarding the expression level of MPP1 gene in several non-erythroid cell lines. (Folia Histochemica et Cytobiologica 2019, Vol. 57, No. 2, 43-55)
\end{abstract}

Key words: membrane palmitoylated protein 1 (MPP1); resting state rafts; lateral membrane organization; raft-associated proteins

\section{Introduction}

The red cell membrane comprises a lipid bilayer with integral membrane proteins embedded in it and a membrane skeleton. During its 120 days life in the circulation the red blood cell undergoes cyclic mechanical shear and deforming stresses in the blood stream, in particular during passages through capillary vessels, which often are four times smaller in diameter than the larger diameter of the erythrocyte. As the lipid bilayer is essentially devoid of extensibility and mechanical resistance (but characterized by high linear elasticity), which results in rapid vesiculation under even mild shear stress, an essential role in

\footnotetext{
Correspondence address: Aleksander F. Sikorski, Professor Department of Cytobiochemistry,

Faculty of Biotechnology,

University of Wroclaw,

F. Joliot-Curie 14a, 50-383 Wroclaw, Poland

e-mail: aleksander.sikorski@uwr.edu.pl
}

maintaining erythrocyte membrane mechanical properties plays membrane skeleton, whose structure and function have been a subject of many studies and reviews [1-3]. The erythrocyte membrane bilayer is not homogeneous; it is characterized by relatively high cholesterol content ( $>20 \%$ lipids by weight) and a set of lipids including sphingolipids (sphingomyelin (SM) and glycolipids) which participate in the generation of membrane lateral heterogeneity by forming domains among which lipid or membrane rafts have been attracting the attention of many laboratories. Literature up to 2014 was surveyed in the previous review [4]. The question remains whether membrane rafts are functional or just remnants of the early stages of nucleated cells. Despite several years of research, there is no great progress towards answering this question. For example, as was mentioned previously [4] there have been several raft-dependent signaling pathways found in red blood cells, e.g. Gs $\alpha$-protein coupled $\beta$-adrenergic receptors cAMP-kinase A leading to the phosphorylation of adducin $[5,6]$. Still the 
erythrocyte membrane remains an interesting object of the study of lateral membrane organization and its function. An example of interesting studies concerns binding of amyloid beta by erythrocyte membrane and its connection with Alzheimer's disease. A fluorescence correlation spectroscopy (FCS)-based study [7] revealed that fluorescently labeled amyloid peptide (A $\beta 1-42)$ localizes to erythrocyte membrane nanodomains which were suggested to be caveolin-rich.

Due to its relative simplicity, erythrocyte membrane still remains a very attractive object to study lateral membrane heterogeneity, i.e. organization of nanodomains. Despite long-term studies we still do not know the molecular mechanisms of how they are organized or even an apparently simpler question, i.e. whether we are dealing with single or multiple kinds of domains in this simple membrane. This issue was addressed by Leonard et al. [8], who via Laurdan-general polarization (GP) studies detected separate nanodomains enriched in SM present in the red blood cell membrane regions of low curvature and cholesterol enriched in the regions of high curvature. Our studies described below focused on one possible mechanism of lateral membrane organization regulation in which peripheral membrane palmitoylated protein 1 (MPP1) was found to play a crucial role, so the considerations presented below concern presentation of the model of this mechanism and address the question of how specific the presented mechanism is, and whether it operates only in blood/erythroid cells or is common in mammals.

\section{Membrane raft concept}

Spatiotemporal organization of biological membranes is characterized by dynamic lateral heterogeneity, as proven by many biophysical and biochemical observations. One of the best known and most acknowledged forms of this heterogeneity is the existence of lateral subcompartments, called membrane rafts (lipid rafts) - a concept formulated by Simons and Ikonen in 1997 [9] which has been the subject of detailed current reviews [10-12].

The basic assemblies, which for clarity in this text will be called resting state rafts, are domains which are highly dynamic $\left(\mathrm{t}_{1 / 2} \sim 1 \mathrm{~s}\right.$ and $\sim 20 \mathrm{~nm}$ in diameter), formed by lo phase lipid and a set of characteristic proteins some of which are permanent residents of these domains (e.g. stomatin and flotillins) while other are temporarily associated with these domains (e.g. growth factor receptors, H-Ras protein, GPI-anchored proteins), which is connected with their activation or resting state. The lipid component of vertebrate cell membrane rafts, enriched in sphingolipids and cholesterol together with other membrane phospholipids which contain long-chain, mostly saturated alkyl residues, is in the so-called liquid ordered phase. Although the molecular basis of lateral phase separation in model membranes seems relatively well understood, available data concerning biological mechanism(s) controlling Ld (liquid disordered) - (liquid ordered) phase separation and formation of rafts in natural membranes are rather scarce and permit only general hypotheses. Lateral interactions of cholesterol with membrane raft-specific lipids seem to be crucial for maintaining these microdomains in the 'lo', liquid-ordered state, which is characterized by decreased conformational (transgauche) freedom and, consequently, reduced "fluidity", compared to the bulk, containing less cholesterol, membrane, which exists in the 'ld' (liquid disordered) state. However, unlike the gel phase in artificial lipid systems they are characterized by similar (within the same range) lateral (translational) mobility to the lo membrane phase, as was measured by FRAP technique on plasma membranes [13-15].

The detergent-resistant membrane (DRM) fraction is defined as a low-density, insoluble in cold nonionic detergent solution membrane fraction floating at $5 / 30 \%$ sucrose step gradient. It was suggested to be useful in assigning potential raft association when changes in the composition are induced by physiologically meaningful events. However, it is possible that DRMs may represent an artificial coalescence of raft proteins and lipids into a residue that does not exist in living cells. Therefore, although there is no simple relationship between DRM and membrane rafts, DRM isolation and characterization is still considered a useful tool, providing some insight into their lateral organization [16, 17].

The molecular basis of Chol:SM complex formation is the hydrogen bond between the amide group of sphingolipid and the hydroxyl group of cholesterol and also the interaction of cholesterol with saturated alkyl chains. Cholesterol is thought to interact with saturated long alkyl chains of other phospholipids. It should be noted that data on lipidomics of the DRMs reveal that, in addition to the abundance of sphingolipids (mostly sphingomyelin), cholesterol and glycolipids, they contain other phospholipids, including some species of PE and PS that mostly contain fully saturated or monounsaturated acyl chains. Predominant among these are the phosphatidylethanolamine glycerophospholipids and plasmalogens [18]. Phosphatidylserine, which is a relatively minor membrane component, is three times more prevalent in the DRM than in the bulk volume of the plasma membrane, while phosphatidylinositols are rather diminished 
within the DRM, as are phosphatidylcholine species. PEs occur in the membrane predominantly as sn-1 saturated, sn-2 unsaturated glycerophospholipids, and experimental data show that some DRM preparations are enriched in 1-stearoyl-2-linoleoyl-sn-glycero-3-phosphoethanolamine (SLPE), regardless of the method of isolation [18]. Our own data indicate that this PE interacts with cholesterol comparably to SM, while dipalmitoyl-PE does not bind cholesterol [19]. This suggests the importance of the structure of acyl chains of particular phospholipids (not all of which are saturated) and also explains the background of the mechanism by which inner-layer phospholipids participate in membrane rafts.

Membrane rafts contain several specific sets of membrane proteins [20], which include membrane proteins belonging to the SPFH family (stomatin/ /prohibitin/flotillin/HflK), such as raft scaffold proteins flotillin-1 and -2, and stomatin or stomatin-like protein. These proteins share a common feature in that they associate with raft domains, possibly through cholesterol binding or oligomerization [21]. Apart from these proteins, and other palmitoylated and transmembrane proteins, membrane rafts also include GPI-anchored proteins and integral and peripheral proteins involved in signal transduction.

Many of the raft proteins are modified with saturated acyl (palmitoyl) chains, or with cholesterol, which is thought to facilitate interactions with lo-domains [22]. The mechanism(s) by which the proteins partition into membrane rafts may involve preferred solubility in the ordered domains and/or chemical affinity for raft lipids, as exemplified by the above-mentioned cholesterol-binding properties of some of the proteins [23]. Other examples include a structural protein motif recognizing sphingolipids or specific glycolipids such as ganglioside [24].

It has long been known that rafts are engaged in cellular signal transmission pathways by hosting a number of receptors and their associated adapter proteins, and also facilitate signaling switches during activation of the respective pathways. These proteins include receptor tyrosine kinases (EGF-R, IGF-1 receptor), non-receptor kinases (e.g. src kinases: Src, Lck, Hck, Fyn, Blk, Lyn, Fgr, Yes, and Yrk [25]), serpentine (G-protein-linked seven transmembrane domain) receptors [26], sigma receptors [27] and the growth factor receptor c-kit, as well as heterotrimeric and monomeric G-proteins and other adaptor proteins. Also endothelial nitric oxide synthase, hedgehog protein and pro- or anti-apoptotic signaling elements have been found in raft domains, as was reviewed [28]. Of particular interest are the potential roles of membrane rafts as signaling platforms in neoplasia
[29] and their possible use as targets for anticancer drugs [28].

The catalogue of physiological functions of rafts involves several important biological processes, and some of them have been shortly described below.

1. Immune signaling involving several innate and adaptive immune responses. These processes have been studied for more than 20 years, beginning with the discovery of involvement of rafts in $\operatorname{IgE}$ signaling [30]. Many components of the immune reactions such as Fcé R1, T cell receptor and B cell receptor move from non-raft (detergent sensitive membrane) to the raft (DRM) upon activation. The DRM fraction was also found to be enriched in signal transduction machinery which includes LCK and FYN kinases and LAT protein and also GPI anchored proteins such as CD14 or THY1 (CD90) [31]. Formation of the so-called immune synapse is considered an example of a "raft signaling platform", a relatively stable structure in diameter in the micrometer range.

2. Host-pathogen interactions. For example, the raft domain is suggested to be a site for HIV budding, binding [32] and stabilization of rafts by cholera toxin (Ctx) and Shiga toxin (Stx) [33, 34]. Also VacA, a vacuolating toxin of $H$. pylori, predominantly associates with the raft phase; however, this binding was found to be independent of oligomerization and pore-forming activity [10, 35]. For example, an interesting observation was published recently indicating that $H$. pylori expression of the cgt gene encoding cholesterol- $\alpha$-glucosyltransferase reduces cholesterol levels in infected gastric epithelial cells, which disrupts membrane rafts, blocking in turn IFN $\gamma$ signaling, allowing bacteria to escape the host inflammatory response [36].

3. In cancer cells, as mentioned above, a considerable number of receptors and adapter proteins involved in signaling pathways engaged in proliferation were found in the DRM fraction, suggesting the crucial role of these structures for signaling of these aberrant cells' development. The literature reviewing various aspects of this subject is particularly rich [27-29]. Some of the signaling pathways and processes could become a target for cancer treatment by using raft domain disrupting agents, as was reviewed previously [28].

4. Involvement in other pathologies. In atherosclerosis, transition of macrophages into foam cells following uptake of ox-LDL is raft dependent as ox-LDL receptors localize to raft domains [37, 38]. Other data [39] suggest that lipid-free apoA-I mediates beneficial effects through attenuation of immune cell membrane raft cholesterol content, 
which affects numerous types of signal transduction pathways that rely on microdomain integrity for assembly and activation. On the other hand, it was found [40] that 7-dehydrocholesterol, but not cholesterol or other sterols, promotes lipid $\mathrm{raft} /$ caveolae formation, leading to suppression of canonical TGF- $\beta$ signaling and atherogenesis.

An important and mostly unresolved question is how the raft domains are organized. In a sense, it predominantly concerns domains corresponding to "resting state rafts" defined by Hancock [41], i.e. functional, more stable domains, $>20 \mathrm{~nm}$ in diameter. The model proposed by Hancock assumes that in cell membranes the smallest nanocomplexes of the so-called membrane rafts precursors, which are built of several protein molecules and lipids, are unstable structures and they disintegrate in milliseconds, but when they are connected in larger structures of approximately $20 \mathrm{~nm}$, they become more stable and functional microdomains and so-called 'resting rafts" [42]. These microdomains can be further associated in structures through protein-protein [43] and protein-lipid interactions with a diameter larger than $100 \mathrm{~nm}$, called signaling platforms. In their stabilization, the membrane or cell skeleton is involved. Recent hypotheses concerning membrane raft organization/ /regulation presented in several reviews [10, 41, 44, 45] include:

1. Lipid-lipid interactions, which include preferential cholesterol binding with sphingolipids [46] and possibly other lipids such as gangliosides [47] or saturated/di- or more unsaturated amino phospholipids [19];

2. Lipid-protein interactions, such as proteins modified by cholesterol [48], e.g. PSD95 [49], MPP1 [4] discussed herein, or by sphingolipid-binding [50] motifs. There are even suggestions that proteins which are secreted from the cells binding $\alpha 2-3$-sialyllactose common in the glycans of monosialogangliosides regulate lateral membrane organization. According to Dalton et al. [51], soluble klotho binding of gangliosides reduces the propensity of membrane to form large ordered domains for endocytosis, which downregulates PI3K signaling.

3. We should add protein-protein interactions, an example of which is MPP1-flotillins and/or possibly other raft residing proteins such as $\mathrm{ABCC} 4$ reported recently by Pitre et al. [52];

4. Hydrophobic match or mismatch; it is hypothetically possible that integral proteins of longer transmembrane domain (TMD) would segregate into lipid domains containing longer fatty acid chains. A solidifying effect of transmembrane peptides of longer TMD and a liquifying effect of shorter TMD peptides were reported previously by Killian et al. [53];

5. Actin-skeleton-driven mechanisms. Cortical actin skeleton is widely considered to affect lateral membrane organization since it has been shown to affect molecular diffusion (hop and trapped diffusion) and supramolecular arrangements in the membrane [54, 55]. Stabilization of nanometer sized domains by an actin filament meshwork was suggested by several groups [56, 57]. Also an interesting approach is a technique of giant plasma membrane vesicles (GPMVs) which provides a model of membrane devoid of membrane/or cortical actin skeleton. This membrane shows phase separation at the micrometer scale [58-60]. Also in the case of erythroid cells liquid-liquid phase separation in the plasma membrane devoid of spectrin-actin skeleton either via extraction with alkaline solution or treatment with latrunculin takes place [61], which somewhat contradicts the statements presented at the beginning of this paragraph. Certainly, keeping the raft domain small $(\sim 20 \mathrm{~nm})$ in most of the resting state cells may be connected with the presence of membrane/cortical actin skeleton, which might be in agreement with the picket-fence model.

In general, involvement of single or multiple protein-protein and or protein-lipid interactions in the organization and regulation of membrane rafts would imply the possibility of regulation at the cellular and/or tissue level. Knowledge of such interactions and their regulation may lead to understanding the molecular mechanism of raft domain organization and regulation and, moreover, may provide ways for manipulation of such processes which would be useful in therapeutic approaches. At this moment, the number of possible proteins engaged in the process of raft domain organization is limited and in the $\mathrm{Pu}$ bMed database we can find only about 6-7 proteins/ /genes which are suspected to be involved in "flat" (non-caveolae) raft domain organization. These are flotillin-1 and -2, annexin A2, C-type lectin domain family 2 member (Clec2i), epithelial membrane protein-2 (Emp2), Pacsin2 (protein kinase $\mathrm{C}$ and casein kinase substrate in neurons 2), raftlin (Rftn1) and calpactin (S100a10).

\section{Membrane palmitoylated protein 1}

Our previous studies [61] on red blood cell rafts led us to the hypothesis that MPP1 and its palmitoylation play a crucial role in lateral membrane organization in erythroid cells. Membrane palmitoylated 


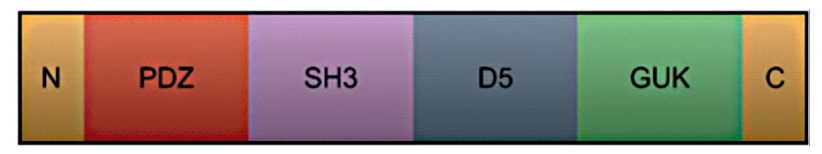

Figure 1. Domain structure of the membrane palmitoylated protein 1 (MPP1). PDZ - postsynaptic density-95/discs large/zonula occludens-1 domain; SH3 - src Homology 3 Domain; D5 - domain contains the binding site for the FERM domain of protein 4.1R; GUK - catalytically inactive guanylate kinase $(\mathrm{GuK})$ domain. Based on data presented in $[61,66]$.

protein 1 (MPP1/p55) encoded by the MPP1 gene belongs to the MAGUK family (membrane associated guanylate kinase homologs) [62, 63]. MPP1 (p55), a MAGUK family member, was first cloned by Ruff et al. [64] and identified as a major target of palmitoylation in red blood cell membrane. It partially fulfills the criteria for scaffolding protein, in that it contains several functional domains which are potentially responsible for simultaneous binding of regulatory and skeletal proteins and is, therefore, an important protein of the membrane skeleton ternary complex (for review see Machnicka et al. [1]). MPP1 shares single GUK, SH3 and PDZ domains and contains a D5/Hook/I3 domain which is responsible for protein 4.1R binding (Fig. 1) [65]. MPP1 has the ability to stabilize mechanical properties of red blood cell membrane by the formation of a tripartite complex with protein 4.1 and glycophorin $C$ linking the spectrin-based membrane skeleton to the membrane bilayer, which is the best known role of MPP1. In this role, the PDZ domain of MPP1 interacts with the cytoplasmic domain of glycophorin C, while the central region (D5- domain) is responsible for interaction with protein 4.1R [65, 66]. This interaction markedly strengthens protein 4.1-glycophorin $\mathrm{C}$ binding [67].

\section{MPP1 is involved in resting state raft formation in erythroid cells}

The functional role of MPP1 palmitoylation is currently not well understood but it is believed that MPP1 palmitoylation is a crucial event involved in raft formation, which might be linked to pathology [4, $28,61]$. Studies performed on erythroid precursor cell line HEL cells revealed that following inhibition of palmitoylation by treatment with the potent protein acetyltransferase (PAT) inhibitor 2-BrP (2-bromo palmitate), the amount of DRM fraction was markedly decreased. When a stable HEL cell-line with silenced expression of the MPP1 gene was used for experiments, the same effect was observed. Moreover, fluorescence lifetime imaging microscopy (FLIM) experiments using di- 4 probe performed on normal and 2-BrP-treated HEL cells and HEL cells with stably silenced $M P P 1$ expression demonstrated a significant decrease in membrane order upon inhibition of palmitoylation, or a decrease in cellular MPP1 level $[61,68]$. The key role of MPP1 protein in the lateral membrane organization has also been demonstrated in model systems which are GPMV vesicles derived from living cells. As a model GPMVs were used, obtained from HEL cells (control and MPP1 KnD) [68]. Numerous studies indicate that the biochemical composition of GPMVs to a significant degree represents their original membranes $[58,69]$ and also reflects the native membrane structure. The results from the research on giant plasma membrane, performed by using several advanced microscopic methods using order-sensing probes, such as C-Laurdan and di-4, showed that, in erythroid cell-membrane derived vesicles, MPP1 plays an important role in maintaining proper order of the membrane [68]. Moreover, the presence of this protein stabilizes the liquid-ordered phase, as observed in the higher miscibility transition temperature of separated vesicles.

In conclusion, changes in membrane fluidity are caused by MPP1, in the absence of major changes in membrane lipid composition, as the fluidity of pure lipids extracted from GPMV membranes remained unchanged and MPP1 recovery in $\mathrm{KnD}$ cells leads to the re-establishment of plasma membrane organization [68]. Furthermore, $M P P 1$ knockdown significantly affects the activation of MAP-kinase signaling via raft-dependent tyrosine kinase receptors, indicating the importance of MPP1 for lateral membrane organization [61]. Further studies showed that, in HEL cells with MPP1 knock-down, the changes in the plasma membrane led to the disruption of signaling cascades from the activated insulin receptor. The signal inhibition occurs at the level of $\mathrm{H}$-Ras, as we did not observe GDP-to-GTP exchange upon insulin treatment [70]. FLIM-FRET microscopy studies revealed impaired interaction of H-Ras with its effector molecule, Raf, in insulin-treated MPP1-knockdowned cells, confirmed by changes in H-Ras lateral diffusion revealed by FRET analysis (Fig. 2). Moreover, in these cells its lateral mobility was not sensitive to insulin treatment. Taken together, our data suggest a relationship between MPP1-dependent plasma-membrane organization and H-Ras activation [70].

\section{MPP1 interacts with different proteins}

As mentioned above, MPP1 forms a tripartite complex with the $4.1 \mathrm{R}$ protein and glycophorin $\mathrm{C}[71,72]$. Pro- 


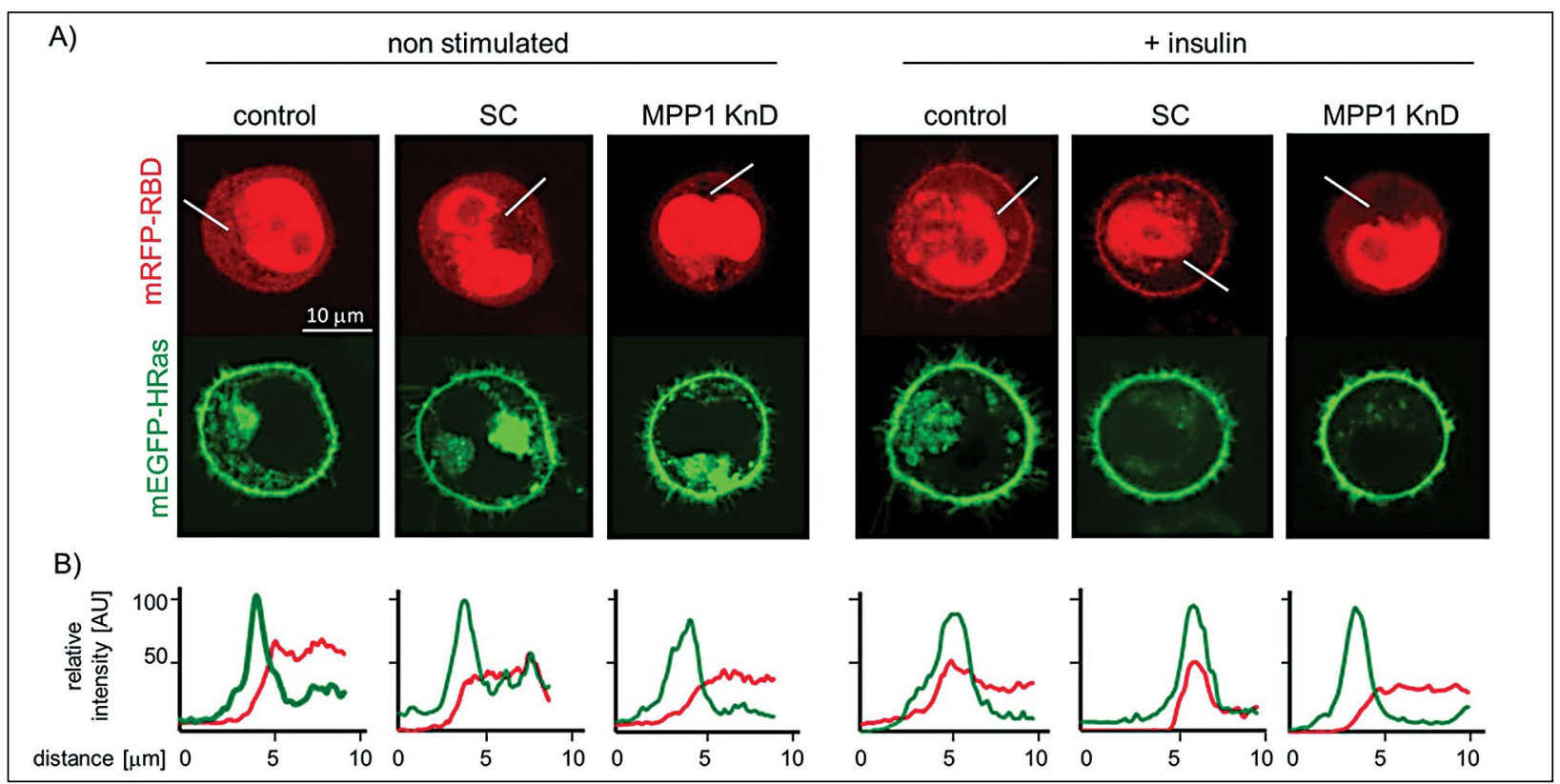

Figure 2. Localization of mEGFP-HRas and mRFP-RBD in non-stimulated and insulin-treated cells. $1 \times 10^{6}$ control, scramble and MPP1 KnD cells were transfected with the appropriate plasmids ( $0.5 \mu \mathrm{g}$ each) using CLB (Lonza) electroporation. After 24 hours, cells were serum-starved for 20 hours and transferred onto glass coverslips coated with $0.1 \mathrm{mg} / \mathrm{ml}$ poly-L-lysine. Cells were observed in a chamber at $37^{\circ} \mathrm{C}$ and $5 \% \mathrm{CO}_{2}$. Photographs were taken before and after addition of insulin [1 $\left.\mu \mathrm{g} / \mathrm{ml}\right]$, $5 \mathrm{~min}$ after stimulation. A. Representative images, scale bar $10 \mu \mathrm{m}$. B. Relative fluorescence intensity profiles revealed impaired recruitment of mRFP-RBD (effector molecule) to the plasma membrane by H-Ras in insulin-treated MPP1 KnD cells (+insulin) — right panel. Reproduced from ref. 70, Podkalicka et al. 2018 (Creative Commons Attribution 3.0 License).

tein 4.1 of red blood cells $(4.1 \mathrm{R})$ is a multifunctional protein that is localized in the membrane skeleton and stabilizes erythrocyte shape and membrane mechanical properties, such as deformability and stability, via lateral interactions with spectrin, actin, glycophorin $\mathrm{C}$ and protein p55 (See reviews: 1, 2, 73). Evidence that MPP1 interacts with protein 4.1 has been shown in the work of Marfatia et al. [72]. Patients with genetic defects resulting in the absence of protein 4.1 (4.1[-] hereditary elliptocytosis) or with defect of glycophorin C (Leach elliptocytosis) also showed absence of MPP1. It indicates that MPP1 is associated, in precise proportions, with the protein 4.1-glycophorin-C complex, linking the skeleton and the membrane. MPP1 contains a binding sequence for the FERM (4.1, ezrin, radixin, moesin) domain of protein 4.1R [74].

Further studies on molecular partners of MPP1 in the erythrocyte membrane by using different methods, such as chemical cross-linking of membrane proteins, co-immunoprecipitation, overlay and solid phase pull-down assays as well as in situ proximity assay, led to the identification of novel interactions of MPP1 and flotillin 1 and flotillin 2 (raft-marker proteins) in the erythrocyte membrane [75]. These interactions are under further, detailed kinetic exploration in a model system and confirm high affinity of formation of these complexes (Olszewska et al. to be published). These interactions were shown to be separate from the above-mentioned well-known protein 4.1-dependent interactions with MPP1 membrane proteins [61]. Flotillins are membrane-associated proteins [76] which are functional in different cellular processes, including clustering of membrane receptors, regulation of signaling pathways [77, 78], participation in cell adhesion [79] and interactions with the cytoskeleton [79]. The mechanism underlying their clustering remains unclear.

Furthermore, it is known that MPP1 interacts with Discs large protein P-dlg (DLG5). P-dlg is a protein encoded by the $P d l g$ gene and is a human homolog of the Drosophila dlg tumor suppressor gene. This protein has three PDZ domains in contrast to MPP1, Dlg-2 and Dlg-3, an SH3 domain and a GUK domain, which are conserved structures in some MAGUK family proteins [80]. Interaction between P-dlg and the GUK domain of MPP1 protein has been confirmed by yeast two-hybrid screening [80]. The C-terminal portion of MPP1 (289-466) has four internal T/S-X-V motifs which may be binding sites for the PDZ domain of P-dlg [80]. It is also interesting that MPP1 is highly 
abundant in AML (acute myeloid leukemia) cells, and interacts with the $\mathrm{ABC}$ transporter, $\mathrm{ABCC} 4$. The studies by Pitre et al. [52] showed that the expression of MPP1 highly correlated with $A B C C 4$ expression in AML, and moreover, it was also associated with poor prognosis for AML patients. The authors carried out an assessment of all PDZ-binding domain proteins on the oligonucleotide microarray with samples from the diagnostic leukemic blasts of 130 pediatric AML patients. They found 81 single PDZ-domain proteins. Analysis (using a correlation with ABCC 4 of $>0.4$ as a threshold) led to the identification of 11 single-PDZ domain proteins that fulfilled the criteria. The MPP1 gene met all requirements with both the highest correlation with ABCC4 $(\mathrm{r}=0.84)$ in the studied AML data sets. Further research carried out by this group also showed that the PDZ domain is necessary and sufficient to bind ABCC4. Furthermore, MPP1 regulates $\mathrm{ABCC} 4$ plasma membrane location and affects stability of ABCC4. Further research of this group indicated that this protein interaction is readily disrupted by a small molecule, antimycin A [52].

The role of MPP1 in non-erythroid cells is understood rather poorly. Mburu et al. [81] have shown that MPP1 forms a complex with whirlin, the protein which binds the Usher protein network in the cochlea and the Crumbs network in the retina, by direct association with USH2A (usherin) and VLGR1 (a member of the 7-transmembrane receptor G-protein, which binds calcium and is expressed in the central nervous system). One of the established physiological roles of MPP1 is its involvement in the regulation of neutrophil polarity. A MPP1 knockout (p55-/-) mouse model [82] showed that, upon agonist stimulation of neutrophils, MPP1 is rapidly recruited to the leading edge. Neutrophils of knockout mouse do not migrate efficiently in vitro and form multiple pseudopods upon chemotactic stimulation, in contrast to normal mouse neutrophils, which form a single pseudopod at the cell front required for efficient chemotaxis. Phosphorylation of Akt is decreased in these cells upon stimulation with chemoattractant, and this appears to be mediated by a PI3K $\gamma$ kinase-independent mechanism [82]. A similar effect was observed in flotillin-1 KO mouse [79], which showed a significant decrease in flotillin 2. Another example is interaction of MPP1 with the FERM domain of NF2 protein. NF2 is a cytoskeletal protein also called merlin, schwannomin or neurofibromin 2 [65]. Neurofibromatosis type 2 (NF2) is a tumor-prone disorder characterized by the development of multiple schwannomas and meningiomas [83]. MPP1-NF2 protein interaction may play a functional role in the regulation of apicobasal polarity and tumor suppression pathways in non-erythroid cells [65].

\section{MPP1 interacts with cholesterol and lipids}

It should be noted that there is also a possibility of MPP1-lipid, mainly cholesterol interactions. We used a simple bioinformatics approach to establish whether MPP1 is capable of binding cholesterol. Modeled and experimentally validated fragment structures were mined from online resources and searched for CRAC and CRAC-like motifs. Several of these motifs were found in the primary structure of MPP1, and these were structurally visualized to see whether they localized to the protein surface. Since all of the CRAC and CRAC-like motifs were found at the surface of MPP1 domains, in silico docking experiments were performed to assess the possibility of interaction between CRAC motifs and cholesterol. The obtained results show that MPP1 can bind cholesterol via CRAC and CRAC-like motifs with moderate to high affinity $\left(\mathrm{K}_{\mathrm{I}}\right.$ in the nano- to micro-molar range). It was also found that palmitoylation-mimicking mutations $(\mathrm{C} / \mathrm{F}$ or $\mathrm{C} / \mathrm{M}$ ) did not affect the affinity of MPP1 towards cholesterol [84]. Further studies on recombinant MPP1 interactions in simple Langmuir monolayer technique revealed that injection of MPP1 into the subphase of an LB monolayer composed of DOPC/SM/Chol (1:1:1 molar ratio) induced an increase in surface pressure, indicating that MPP1 molecules were incorporated into the lipid monolayer. The compressibility modulus isotherms of MPP1, lipids and lipid-MPP1 films have essentially different shapes from one another. In addition, this interaction was found to be sensitive to the presence of cholesterol in the lipid monolayer, as adding MPP1 to the subphase of lipid monolayers containing cholesterol resulted in a much larger increase in surface area than when MPP1 is injected into the subphase of a lipid monolayer devoid of cholesterol [85]. Moreover, MPP1-bound cholesterol was found to competitively inhibit surface pressure changes of the phospholipid monolayer [86].

\section{MPP1 is the resting state raft organizing protein in erythroid cells}

In conclusion, we propose MPP1 to be a main component of the mechanisms responsible for lateral organization of the erythroid cell membrane. The studies presented above indicate a new role for MPP1, and present a novel linkage between membrane raft organization and their function.

The molecular mechanism underlying MPP1's function as a resting state raft organizer remains to be explored. Our hypothesis is that, upon palmitoylation, the affinity of MPP1 for binding to the pre-existing nanocluster membrane increases. As preexisting na- 


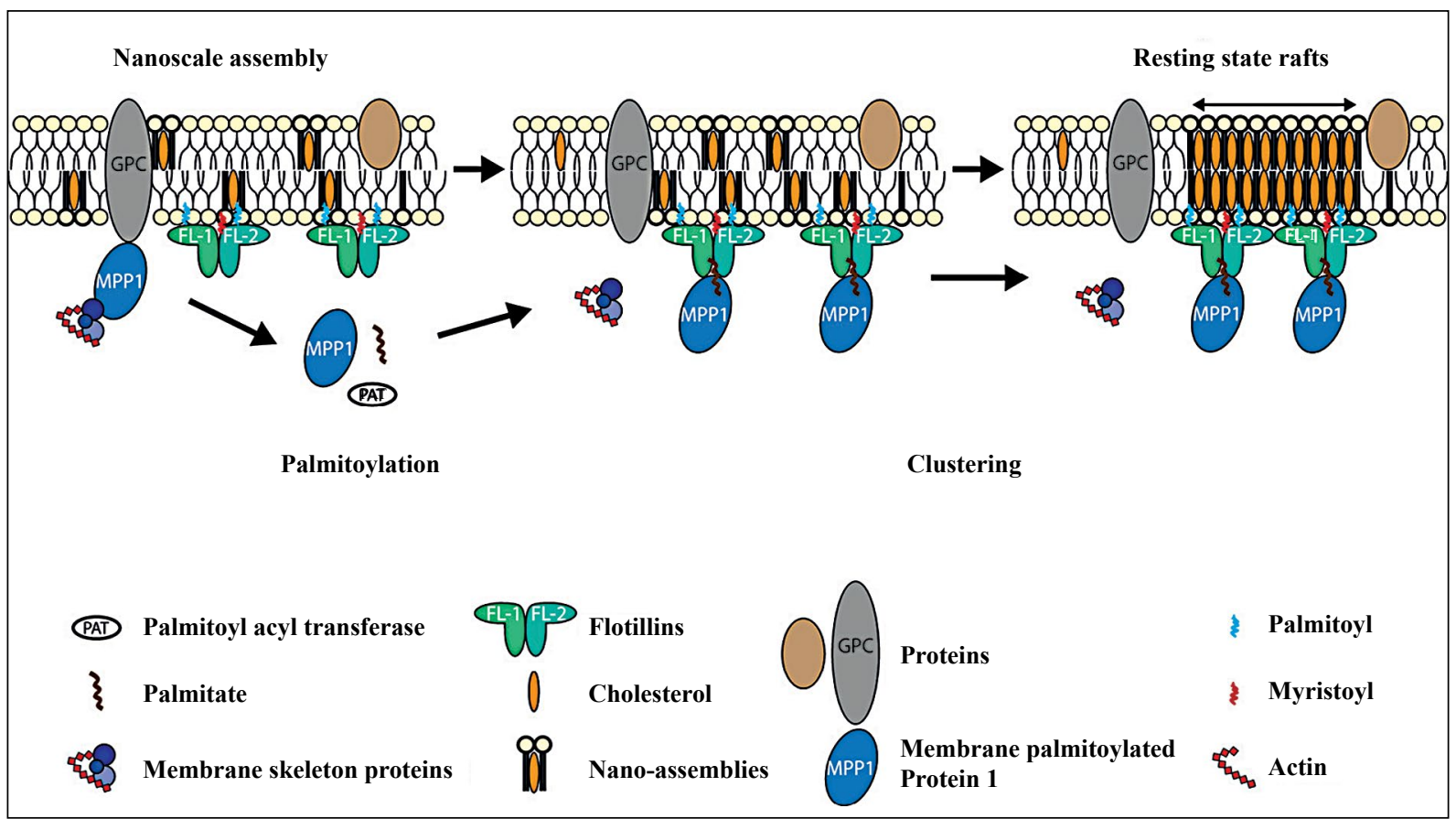

Figure 3. Schematic model of the proposed mechanism of resting state membrane raft organization in erythroid cells. Free MPP1 which is in equilibrium with membrane skeletally associated pool of this protein binds to the pre-existing short-lived flotillins-based nano-assemblies $(<10 \mathrm{~nm}$ in diameter, half time $<0.1 \mathrm{~ms})$. This leads to oligomerization and co-assembly of flotillin-based nanoclusters into larger-scale complexes and formation of a more stable (half time $\sim 1 \mathrm{~s})$ larger $(>20 \mathrm{~nm}$ ) structures called "resting state rafts" in erythroid cell membrane. Modified from Biernatowska et al. [75]) (CC BY-NC-ND 4.0).

noclusters we consider protein-cholesterol/lipid complexes corresponding to short-lived (half time $<0.1 \mathrm{~ms}$ ) nanocomplexes ( $<10 \mathrm{~nm}$ in diameter), described previously by others [87-89]. This suggestion is based on the observations that in HEL cells, upon inhibition of palmitoylation, all MPP1 remains in the high-density "skeletal" fraction within the density-gradient profile of the DRM, and also MPP1 KnD Hel cell membrane is characterized by increased membrane fluidity as measured by FLIM of the D- 4 probe. We believe that flotillin-1 and -2 are important components of these nanoclusters, which was confirmed by the fact that flotillins are found in the DRM residual fraction even after treatment with beta methyl cyclodextrin. Binding of palmitoylated MPP1 to the pre-existing nanocomplexes via flotillins and perhaps via cholesterol-binding regions as was recently documented [75, 84-86] induces their fusion and stabilizes them as membrane resting-state rafts, which are larger $(\sim 20 \mathrm{~nm})$ in diameter [90], more stable ( $\sim 1 \mathrm{~s})$, detergent-resistant, and which become functional (see Fig. 3).

\section{MPP1 is not the raft organizer in most cells in mammalian organisms}

As the data on the level of MPP1 protein in the various cell types of the human body are only fragmentary (e.g. https://www.ncbi.nlm.nih.gov/ /gene/4354), we decided to test the presence of MPP1 in several human cell types. First of all we completed screening through blood cells. Cells were isolated from whole blood on the density gradient Ficoll-Paque (density $1.077 \mathrm{~g} / \mathrm{mL}$ ) and then further purified using MicroBeads by Miltenyi Biotec. The level of MPP1 gene expression in the studied cells was assessed by Western blot analysis and RT-PCR (reverse transcription). Additionally, in some cases the effect of palmitoylation on the formation of DRM fractions was tested on these cells (Table 1). For this purpose, a palmitoylation inhibitor, 2-BrP, was used. The DRM fraction was isolated via cold $1 \%$ Triton X-100 extraction and ultracentrifugation of the extract on a sucrose step (40/30/5\%) gradient. Literature data indicated the presence MPP1 in erythroid cells, mouse granulocytes and several myeloid and lymphoid cell lines. In our experience high expression of MPP1 could be observed in most blood cells and blood cell-derived neoplastic cell lines. As could be anticipated, a relatively high level of MPP1 could be detected in the platelets as their parental cell has a common precursor with erythrocytes. In the blood cells the amount of DRM fraction protein and cholesterol and first of all the presence of MPP1 in the DRM appeared to be sensitive to inhibition 
Table 1. The presence of MPP1 protein or its mRNA in different cell types

\begin{tabular}{|c|c|c|c|c|c|}
\hline Cells/cell line & $\begin{array}{l}\text { MPP1 (Western } \\
\text { blotting) }\end{array}$ & $\begin{array}{c}\text { MPP1 } \\
\text { (rtPCR) }\end{array}$ & DRM & $\begin{array}{c}\text { DRM sensitivity to } 2-\mathrm{BrP} \\
\text { treatment }\end{array}$ & $\begin{array}{l}\text { Changes of fluorescence } \\
\text { life-time of di-4 probe }\end{array}$ \\
\hline Erythrocytes & +++ & $+++^{A}$ & +++ & +++ & +++ \\
\hline HEL & +++ & +++ & +++ & +++ & +++ \\
\hline K562 & +++ & +++ & +++ & +++ & nd \\
\hline Thrombocytes & +++ & +++ & nd & nd & nd \\
\hline Monocytes & +++ & +++ & nd & nd & nd \\
\hline Neutrophils & +++ & +++ & nd & nd & nd \\
\hline Jurkat T & +++ & nd & +++ & +++ & +++ \\
\hline HL60 & $+/-$ & nd & nd & nd & nd \\
\hline HEK293 & ++ & nd & -- & -- & nd \\
\hline MDA-MB-231 & $+/-$ & + & - & - & nd \\
\hline HeLa & - & - & nd & nd & nd \\
\hline A549 & - & - & nd & nd & nd \\
\hline A375 & - & - & nd & nd & nd \\
\hline SCOV3 & - & - & nd & nd & nd \\
\hline MCF7 & - & - & nd & nd & nd \\
\hline DU145 & - & - & nd & nd & nd \\
\hline
\end{tabular}

${ }^{A}$ Reticulocyte +++ present; - absent; nd - not determined. The localization in DRM fractions and/or sensitivity to 2-BrP treatment are also presented. Cells or cell lines were cultured in standard conditions. For Western blot analyses, cells were dissolved in Laemmli buffer and subjected to SDS-PAGE, transferred onto nitrocellulose filter, blocked with 5\% milk proteins and probed with anti-MPP1 rabbit antibodies (Aviva Systems Biology, San Diego, CA, USA) and developed with appropriate secondary antibody conjugated to horseradish peroxidase in a chemiluminescent reaction. Reverse transcriptase (RT) reaction was performed using isolated total RNA (miRNeasy kit QIAGEN, Hilden, Germany) translated into cDNA via RT reaction (RevertAid First Strand cDNA Synthesis Kit Thermo Fisher Scientific, Waltham, MA, USA). Primers for MPP1 (Genomed, Warsaw, Poland; FP: CCTACGAGGAAGTCGTTCGG, RP: GGTCTGGGGCTCAATGTCAA, 340 bp, and for beta-actin Thermo Fisher Scientific: FP; TACAATGAGCTGCGTGTGGCTCCCG, RP: AATGGTGATGACCTGGCCTGGCCGTCAGGC, 540 bp) were used.

of palmitoylation with 2-BrP (Table 1) as was the fluidity of the cell membrane in vivo, as measured via FLIM analysis of the di-4 probe. However, to our surprise, the MPP1 gene was not expressed to high levels in studied cells of human tumor-derived cell lines apart from HEK293 (human embryonic kidney). In the latter cells an easily detectable signal was observed via either RT-PCR or Western blot, although dependence upon palmitoylation of DRM localization of MPP1 could not be observed in the applied experimental conditions. We have to keep in mind that the remaining cell lines are derived from human tumors, so we cannot anticipate with a high degree of certainty that in the case of normal tissues the results would be the same. In the case of blood cells most of the neoplastic cells expressed the MPP1 gene at a high level, but in some of them (promyelocytic leukemia HL60 or Raji Burkitt's lymphoma) MPP1 could not be detected in Western blot.

The obtained results clearly show that MPP1 is expressed at a negligible level in many types of human cancer epithelial cells, in contrast to cells of erythroid origin, where the level of their expression is high. The results support the hypothesis that the MPP1 protein is not responsible for the formation and maintenance of membrane rafts in epithelial cells, and this may suggest a different model of formation of these domains than in the erythroid cells.

\section{Conclusions}

The data collected by us clearly indicate that MPP1 participates in the resting state membrane rafts' organization and regulation at least in erythroid cells. Summarizing collected information about membrane raft formation and the role of MPP1, we conclude that a network of proteins involving flotillins and flotillin-binding proteins is responsible for bringing lipids closely enough to form small, relatively stable $(\sim 1 \mathrm{~s})$ lo phase domains which form resting-state rafts which are functional in the sense of recruiting appropriate activated (or becoming activated after becoming a part of the raft) receptors and other elements of the signaling pathway. It may also facilitate dissociation 
of certain elements of the signaling pathway from the raft domain upon their activation, e.g. H-Ras dissociating from the raft domain after exchange of GDP for GTP. Effects of MPP1 knock-out on neutrophil polarization or interaction with $\mathrm{ABBC} 4$, which causes a significant increase in cell resistance to chemotherapy, may also be connected with MPP1's resting state raft organizing role.

Our preliminary results indicate that the MPP1 protein is abundant only in blood cells and their precursors, in other cells such as HEK293 there is a smaller amount of this protein, whereas in breast cancer cells such as MDA-MB-231 we observed only trace amounts of MPP1. The data collected by us may suggest that there may be more than one mechanism of the formation of membrane rafts. Our hypothesis assumes that another MAGUK protein or even proteins which do not belong to the MAGUK protein family may perform a similar function as MPP1. For example, a similar function was recently suggested for the palmitoylation of Rac1 in COS-7 and MEF cells [91], and these authors implicate a role for the actin cytoskeleton in the mechanism of raft clustering in these cells. Therefore, the answer to the question posed by the title at this stage of research is that the MPP1-based molecular mechanism of resting state raft domain organization is a mechanism limited to certain types of cells, and the question of such domain organization in other cells remains open. However, we anticipate that it could be a flotillin-binding protein based mechanism.

\section{References}

1. Machnicka B, Czogalla A, Hryniewicz-Jankowska A, et al. Spectrins: a structural platform for stabilization and activation of membrane channels, receptors and transporters. Biochim Biophys Acta. 2014; 1838(2): 620-634, doi: 10.1016/j.bbamem.2013.05.002, indexed in Pubmed: 23673272.

2. Machnicka B, Grochowalska R, Bogusławska DM, et al. Spectrin-based skeleton as an actor in cell signaling. Cell Mol Life Sci. 2012; 69(2): 191-201, doi: 10.1007/s00018-011-0804-5, indexed in Pubmed: 21877118.

3. Lux SE. Anatomy of the red cell membrane skeleton: unanswered questions. Blood. 2016; 127(2): 187-199, doi: 10.1182/ blood-2014-12-512772, indexed in Pubmed: 26537302.

4. Sikorski AF, Podkalicka J, Jones W, et al. Membrane rafts in the erythrocyte membrane: a novel role of MPP1p55. Adv Exp Med Biol. 2015; 842: 61-78, doi: 10.1007/978-3-319-11280-0_5, indexed in Pubmed: 25408337.

5. Koshino I, Takakuwa Y. Disruption of lipid rafts by lidocaine inhibits erythrocyte invasion by Plasmodium falciparum. Exp Parasitol. 2009; 123(4): 381-383, doi: 10.1016/j.exppara.2009.08.019, indexed in Pubmed: 19733566.

6. Kamata K, Manno S, Ozaki M, et al. Functional evidence for presence of lipid rafts in erythrocyte membranes: Gsalpha in rafts is essential for signal transduction. Am J Hematol. 2008; 83(5): 371-375, doi: 10.1002/ajh.21126, indexed in Pubmed: 18181202 .
7. Hashimoto M, Hossain S, Katakura M, et al. The binding of A $\beta 1-42$ to lipid rafts of RBC is enhanced by dietary docosahexaenoic acid in rats: Implicates to Alzheimer's disease. Biochim Biophys Acta. 2015; 1848(6): 1402-1409, doi: 10.1016/j. bbamem.2015.03.008, indexed in Pubmed: 25782726.

8. Leonard C, Conrard L, Guthmann M, et al. Contribution of plasma membrane lipid domains to red blood cell (re) shaping. Sci Rep. 2017; 7(1): 4264, doi: 10.1038/s41598-017-04388-z.

9. Simons K, Ikonen E. Functional rafts in cell membranes. Nature. 1997; 387(6633): 569-572, doi: 10.1038/42408, indexed in Pubmed: 9177342.

10. Raghunathan K, Kenworthy AK. Dynamic pattern generation in cell membranes: Current insights into membrane organization. Biochim Biophys Acta Biomembr. 2018; 1860(10): 2018-2031, doi: 10.1016/j.bbamem.2018.05.002, indexed in Pubmed: 29752898.

11. Bieberich E. Sphingolipids and lipid rafts: Novel concepts and methods of analysis. Chem Phys Lipids. 2018; 216: 114-131, doi: 10.1016/j.chemphyslip.2018.08.003, indexed in Pubmed: 30194926.

12. Kinoshita M, Suzuki KGN, Murata M, et al. Evidence of lipid rafts based on the partition and dynamic behavior of sphingomyelins. Chem Phys Lipids. 2018; 215: 84-95, doi: 10.1016/j. chemphyslip.2018.07.002, indexed in Pubmed: 30005889.

13. Rawat SS, Zimmerman C, Johnson BT, et al. Restricted lateral mobility of plasma membrane CD4 impairs HIV-1 envelope glycoprotein mediated fusion. Mol Membr Biol. 2008; 25(1): 83-94, doi: 10.1080/09687680701613713, indexed in Pubmed: 18097956.

14. Pucadyil TJ, Chattopadhyay A. Effect of cholesterol on lateral diffusion of fluorescent lipid probes in native hippocampal membranes. Chem Phys Lipids. 2006; 143(1-2): 11-21, doi: 10.1016/j.chemphyslip.2006.04.003, indexed in Pubmed: 16797513.

15. Simons K, Vaz WLC. Model systems, lipid rafts, and cell membranes. Annu Rev Biophys Biomol Struct. 2004; 33: 269-295, doi: 10.1146/annurev.biophys.32.110601.141803, indexed in Pubmed: 15139814.

16. Lingwood D, Simons K. Detergent resistance as a tool in membrane research. Nat Protoc. 2007; 2(9): 2159-2165, doi: 10.1038/nprot.2007.294, indexed in Pubmed: 17853872.

17. Lichtenberg D, Goñi FM, Heerklotz H. Detergent-resistant membranes should not be identified with membrane rafts. Trends Biochem Sci. 2005; 30(8): 430-436, doi: 10.1016/j. tibs.2005.06.004, indexed in Pubmed: 15996869.

18. Pike LJ, Han X, Gross RW. Epidermal Growth Factor Receptors Are Localized to Lipid Rafts. J Biol Chem. 2005; 280(29): 26796-26804, doi: 10.1074/jbc.M503805200, indexed in Pubmed: 15917253.

19. Grzybek M, Kubiak J, Łach A, et al. A raft-associated species of phosphatidylethanolamine interacts with cholesterol comparably to sphingomyelin. A Langmuir-Blodgett monolayer study. PLoS One. 2009; 4(3): e5053, doi: 10.1371/journal. pone.0005053, indexed in Pubmed: 19330037.

20. Brown DA, London E. Structure and function of sphingolipid- and cholesterol-rich membrane rafts. J Biol Chem. 2000; 275(23): 17221-17224, doi: 10.1074/jbc.R000005200, indexed in Pubmed: 10770957.

21. Browman DT, Hoegg MB, Robbins SM. The SPFH domain-containing proteins: more than lipid raft markers. Trends Cell Biol. 2007; 17(8): 394-402, doi: 10.1016/j. tcb.2007.06.005, indexed in Pubmed: 17766116.

22. Wang TY, Leventis R, Silvius JR. Fluorescence-based evaluation of the partitioning of lipids and lipidated peptides into 
liquid-ordered lipid microdomains: a model for molecular partitioning into. Biophys J. 2000; 79(2): 919-933, doi: 10.1016/ S0006-3495(00)76347-8, indexed in Pubmed: 10920023.

23. Fantini J, Barrantes FJ. How cholesterol interacts with membrane proteins: an exploration of cholesterol-binding sites including CRAC, CARC, and tilted domains. Front Physiol. 2013; 4: 31, doi: 10.3389/fphys.2013.00031, indexed in $\mathrm{Pu}-$ bmed: 23450735.

24. Mahfoud R, Garmy N, Maresca M, et al. Identification of a common sphingolipid-binding domain in Alzheimer, prion, and HIV-1 proteins. J Biol Chem. 2002; 277(13): 11292-11296, doi: 10.1074/jbc.M111679200, indexed in Pubmed: 11792705.

25. Boggon TJ, Eck MJ. Structure and regulation of Src family kinases. Oncogene. 2004; 23(48): 7918-7927, doi: 10.1038/ sj.onc.1208081, indexed in Pubmed: 15489910.

26. Pike L. Lipid rafts. Journal of Lipid Research. 2003; 44(4): 655-667, doi: 10.1194/jlr.r200021-jlr200.

27. Aydar E, Palmer CP, Djamgoz MBA. Sigma receptors and cancer: possible involvement of ion channels. Cancer Res. 2004; 64(15): 5029-5035, doi: 10.1158/0008-5472.CAN-032329, indexed in Pubmed: 15289298.

28. Hryniewicz-Jankowska A, Augoff K, Biernatowska A, et al. Membrane rafts as a novel target in cancer therapy. Biochim Biophys Acta. 2014; 1845(2): 155-165, doi: 10.1016/j.bbcan.2014.01.006, indexed in Pubmed: 24480320.

29. Staubach S, Hanisch FG. Lipid rafts: signaling and sorting platforms of cells and their roles in cancer. Expert Rev Proteomics. 2011; 8(2): 263-277, doi: 10.1586/epr.11.2, indexed in Pubmed: 21501018.

30. Field KA, Holowka D, Baird B. Fc epsilon RI-mediated recruitment of p53/56lyn to detergent-resistant membrane domains accompanies cellular signaling. Proc Natl Acad Sci U S A. 1995; 92(20): 9201-9205, doi: 10.1073/pnas.92.20.9201, indexed in Pubmed: 7568101.

31. Varshney P, Yadav V, Saini N. Lipid rafts in immune signalling: current progress and future perspective. Immunology. 2016; 149(1): 13-24, doi: 10.1111/imm.12617, indexed in Pubmed: 27153983.

32. Dick RA, Goh SL, Feigenson GW, et al. HIV-1 Gag protein can sense the cholesterol and acyl chain environment in model membranes. Proc Natl Acad Sci U S A. 2012; 109(46): 18761-18766, doi: 10.1073/pnas.1209408109, indexed in $\mathrm{Pu}-$ bmed: 23010924.

33. Chinnapen DJF, Chinnapen H, Saslowsky D, et al. Rafting with cholera toxin: endocytosis and trafficking from plasma membrane to ER. FEMS Microbiol Lett. 2007; 266(2): 129-137, doi: 10.1111/j.1574-6968.2006.00545.x, indexed in Pubmed: 17156122.

34. Sandvig K, Bergan J, Kavaliauskiene S, et al. Lipid requirements for entry of protein toxins into cells. Prog Lipid Res. 2014; 54: 1-13, doi: 10.1016/j.plipres.2014.01.001, indexed in Pubmed: 24462587.

35. Raghunathan K, Foegeding NJ, Campbell AM, et al. Determinants of Raft Partitioning of the Helicobacter pylori Pore-Forming Toxin VacA. Infect Immun. 2018; 86(5), doi: 10.1128/IAI.00872-17, indexed in Pubmed: 29531133.

36. Morey P, Pfannkuch L, Pang E, et al. Helicobacter pylori Depletes Cholesterol in Gastric Glands to Prevent Interferon Gamma Signaling and Escape the Inflammatory Response. Gastroenterology. 2018; 154(5): 1391-1404.e9, doi: 10.1053/j. gastro.2017.12.008, indexed in Pubmed: 29273450.

37. Rios FJO, Ferracini M, Pecenin M, et al. Uptake of oxLDL and IL-10 production by macrophages requires PAFR and CD36 recruitment into the same lipid rafts. PLoS One. 2013;
8(10): e 76893, doi: 10.1371/journal.pone.0076893, indexed in Pubmed: 24130805.

38. Rios FJ, Koga MM, Pecenin M, et al. Oxidized LDL induces alternative macrophage phenotype through activation of CD36 and PAFR. Mediators Inflamm. 2013; 2013: 198193, doi: 10.1155/2013/198193, indexed in Pubmed: 24062612.

39. Kaul S, Xu H, Zabalawi M, et al. Lipid-Free Apolipoprotein A-I Reduces Progression of Atherosclerosis by Mobilizing Microdomain Cholesterol and Attenuating the Number of CD131 Expressing Cells: Monitoring Cholesterol Homeostasis Using the Cellular Ester to Total Cholesterol Ratio. J Am Heart Assoc. 2016; 5(11), doi: 10.1161/JAHA.116.004401, indexed in Pubmed: 27821400.

40. Huang SS, Liu IH, Chen CL, et al. 7-Dehydrocholesterol (7DHC), But Not Cholesterol, Causes Suppression of Canonical TGF- $\beta$ Signaling and Is Likely Involved in the Development of Atherosclerotic Cardiovascular Disease (ASCVD). J Cell Biochem. 2017; 118(6): 1387-1400, doi: 10.1002/jcb.25797, indexed in Pubmed: 27862220.

41. Hancock JF. Lipid rafts: contentious only from simplistic standpoints. Nat Rev Mol Cell Biol. 2006; 7(6): 456-462, doi: 10.1038/nrm1925, indexed in Pubmed: 16625153.

42. Simons K, Gerl MJ. Revitalizing membrane rafts: new tools and insights. Nat Rev Mol Cell Biol. 2010; 11(10): 688-699, doi: 10.1038/nrm2977, indexed in Pubmed: 20861879.

43. Pike LJ. Rafts defined: a report on the Keystone Symposium on Lipid Rafts and Cell Function. J Lipid Res. 2006; 47(7): 1597-1598, doi: 10.1194/jlr.E600002-JLR200, indexed in Pubmed: 16645198.

44. Sezgin E, Levental I, Mayor S, et al. The mystery of membrane organization: composition, regulation and roles of lipid rafts. Nat Rev Mol Cell Biol. 2017; 18(6): 361-374, doi: 10.1038/ nrm.2017.16, indexed in Pubmed: 28356571.

45. Sevcsik E, Schütz GJ. With or without rafts? Alternative views on cell membranes. Bioessays. 2016; 38(2): 129-139, doi: 10.1002/bies.201500150, indexed in Pubmed: 26666984

46. Ramstedt B, Slotte JP. Interaction of cholesterol with sphingomyelins and acyl-chain-matched phosphatidylcholines: a comparative study of the effect of the chain length. Biophys J. 1999; 76(2): 908-915, doi: 10.1016/S0006-3495(99)77254-1, indexed in Pubmed: 9929492.

47. Lozano MM, Hovis JS, Moss FR, et al. Dynamic Reorganization and Correlation among Lipid Raft Components. J Am Chem Soc. 2016; 138(31): 9996-10001, doi: 10.1021/ jacs.6b05540, indexed in Pubmed: 27447959.

48. Schwarzer R, Levental I, Gramatica A, et al. The cholesterol-binding motif of the HIV-1 glycoprotein gp41 regulates lateral sorting and oligomerization. Cell Microbiol. 2014; 16(10): 1565-1581, doi: 10.1111/cmi.12314, indexed in Pubmed: 24844300 .

49. Tulodziecka K, Diaz-Rohrer BB, Farley MM, et al. Remodeling of the postsynaptic plasma membrane during neural development. Mol Biol Cell. 2016; 27(22): 3480-3489, doi: 10.1091/mbc.E16-06-0420, indexed in Pubmed: 27535429.

50. Contreras FX, Ernst AM, Haberkant P, et al. Molecular recognition of a single sphingolipid species by a protein's transmembrane domain. Nature. 2012; 481(7382): 525-529, doi: 10.1038/nature10742, indexed in Pubmed: 22230960.

51. Dalton G, An SW, Al-Juboori SI, et al. Soluble klotho binds monosialoganglioside to regulate membrane microdomains and growth factor signaling. Proc Natl Acad Sci U S A. 2017; 114(4): 752-757, doi: 10.1073/pnas.1620301114, indexed in Pubmed: 28069944. 
52. Pitre A, Ge Y, Lin W, et al. An unexpected protein interaction promotes drug resistance in leukemia. Nat Commun. 2017; 8(1): 1547, doi: 10.1038/s41467-017-01678-y, indexed in Pubmed: 29146910.

53. Killian JA, von Heijne G. How proteins adapt to a membrane-water interface. Trends Biochem Sci. 2000; 25(9): 429-434, doi: 10.1016/S0968-0004(00)01626-1, indexed in Pubmed: 10973056.

54. Köster DV, Mayor S. Cortical actin and the plasma membrane: inextricably intertwined. Curr Opin Cell Biol. 2016; 38: 81-89, doi: 10.1016/j.ceb.2016.02.021, indexed in Pubmed: 26986983.

55. Marguet D, Lenne PF, Rigneault H, et al. Dynamics in the plasma membrane: how to combine fluidity and order. EMBO J. 2006; 25(15): 3446-3457, doi: 10.1038/sj.emboj.7601204, indexed in Pubmed: 16900097.

56. Honigmann A, Sadeghi S, Keller J, et al. A lipid bound actin meshwork organizes liquid phase separation in model membranes. Elife. 2014; 3: e01671, doi: 10.7554/eLife.01671, indexed in Pubmed: 24642407.

57. Ehrig J, Petrov EP, Schwille P. Near-critical fluctuations and cytoskeleton-assisted phase separation lead to subdiffusion in cell membranes. Biophys J. 2011; 100(1): 80-89, doi: 10.1016/j. bpj.2010.11.002, indexed in Pubmed: 21190659.

58. Levental I, Grzybek M, Simons K. Raft domains of variable properties and compositions in plasma membrane vesicles. Proc Natl Acad Sci U S A. 2011; 108(28): 11411-11416, doi: 10.1073/pnas.1105996108, indexed in Pubmed: 21709267.

59. Baumgart T, Hammond AT, Sengupta P, et al. Large-scale fluid/fluid phase separation of proteins and lipids in giant plasma membrane vesicles. Proc Natl Acad Sci U S A. 2007; 104(9): 3165-3170, doi: 10.1073/pnas.0611357104, indexed in Pubmed: 17360623.

60. Sezgin E, Kaiser HJ, Baumgart T, et al. Elucidating membrane structure and protein behavior using giant plasma membrane vesicles. Nat Protoc. 2012; 7(6): 1042-1051, doi: 10.1038/ nprot.2012.059, indexed in Pubmed: 22555243.

61. Biernatowska A, Podkalicka J, Majkowski M, et al. The role of MPP1/p55 and its palmitoylation in resting state raft organization in HEL cells. Biochim Biophys Acta. 2013; 1833(8): 1876-1884, doi: 10.1016/j.bbamcr.2013.03.009, indexed in Pubmed: 23507198.

62. Dimitratos SD, Woods DF, Stathakis DG, et al. Signaling pathways are focused at specialized regions of the plasma membrane by scaffolding proteins of the MAGUK family. Bioessays. 1999; 21(11): 912-921, doi: 10.1002/(SICI)15211878(199911)21:11<912::AID-BIES3>3.0.CO;2-Z, indexed in Pubmed: 10517864.

63. Anderson JM. Cell signalling: MAGUK magic. Curr Biol. 1996; 6(4): 382-384, indexed in Pubmed: 8723338.

64. Ruff P, Speicher DW, Husain-Chishti A. Molecular identification of a major palmitoylated erythrocyte membrane protein containing the src homology 3 motif. Proc Natl Acad Sci U S A. 1991; 88(15): 6595-6599, doi: 10.1073/pnas.88.15.6595, indexed in Pubmed: 1713685.

65. Seo PS, Quinn BJ, Khan AA, et al. Identification of erythrocyte p55/MPP1 as a binding partner of NF2 tumor suppressor protein/Merlin. Exp Biol Med (Maywood). 2009; 234(3): 255-262, doi: 10.3181/0809-RM-275, indexed in Pubmed: 19144871.

66. Marfatia SM, Morais-Cabral JH, Kim AC, et al. The PDZ domain of human erythrocyte p55 mediates its binding to the cytoplasmic carboxyl terminus of glycophorin C. Analysis of the binding interface by in vitro mutagenesis. J Biol Chem.
1997; 272(39): 24191-24197, doi: 10.1074/jbc.272.39.24191, indexed in Pubmed: 9305870.

67. Hemming NJ, Anstee DJ, Staricoff MA, et al. Identification of the membrane attachment sites for protein 4.1 in the human erythrocyte. J Biol Chem. 1995; 270(10): 5360-5366, doi: 10.1074/jbc.270.10.5360, indexed in Pubmed: 7890649.

68. Podkalicka J, Biernatowska A, Majkowski M, et al. MPP1 as a Factor Regulating Phase Separation in Giant Plasma Membrane-Derived Vesicles. Biophys J. 2015; 108(9): 2201-2211, doi: 10.1016/j.bpj.2015.03.017, indexed in Pubmed: 25954878.

69. Levental I, Lingwood D, Grzybek M, et al. Palmitoylation regulates raft affinity for the majority of integral raft proteins. Proc Natl Acad Sci U S A. 2010; 107(51): 22050-22054, doi: 10.1073/pnas.1016184107, indexed in Pubmed: 21131568.

70. Podkalicka J, Biernatowska A, Olszewska P, et al. The microdomain-organizing protein MPP1 is required for insulin-stimulated activation of H-Ras. Oncotarget. 2018; 9(26): 18410-18421, doi: 10.18632/oncotarget.24847, indexed in Pubmed: 29719614.

71. Chishti AH. Function of p55 and its nonerythroid homologues. Curr Opin Hematol. 1998; 5(2): 116-121, indexed in Pubmed: 9570704.

72. Marfatia SM, Lue RA, Branton D, et al. In vitro binding studies suggest a membrane-associated complex between erythroid p55, protein 4.1, and glycophorin C. J Biol Chem. 1994; 269(12): 8631-8634, indexed in Pubmed: 8132590.

73. Diakowski W, Grzybek M, Sikorski AF. Protein 4.1, a component of the erythrocyte membrane skeleton and its related homologue proteins forming the protein 4.1/FERM superfamily. Folia Histochem Cytobiol. 2006; 44(4): 231-248, indexed in Pubmed: 17219717.

74. Ward RE, Schweizer L, Lamb RS, et al. The protein 4.1, ezrin, radixin, moesin (FERM) domain of Drosophila Coracle, a cytoplasmic component of the septate junction, provides functions essential for embryonic development and imaginal cell proliferation. Genetics. 2001; 159(1): 219-228, indexed in Pubmed: 11560899.

75. Biernatowska A, Augoff K, Podkalicka J, et al. MPP1 directly interacts with flotillins in erythrocyte membrane - Possible mechanism of raft domain formation. Biochim Biophys Acta Biomembr. 2017; 1859(11): 2203-2212, doi: 10.1016/j.bbamem.2017.08.021, indexed in Pubmed: 28865798.

76. Lang DM, Lommel S, Jung M, et al. Identification of reggie-1 and reggie-2 as plasmamembrane-associated proteins which cocluster with activated GPI-anchored cell adhesion molecules in non-caveolar micropatches in neurons. J Neurobiol. 1998; 37(4): 502-523, indexed in Pubmed: 9858255.

77. Neumann-Giesen C, Fernow I, Amaddii M, et al. Role of EGF-induced tyrosine phosphorylation of reggie-1/flotillin-2 in cell spreading and signaling to the actin cytoskeleton. J Cell Sci. 2007; 120(Pt 3): 395-406, doi: 10.1242/jcs.03336, indexed in Pubmed: 17213334.

78. Amaddii M, Meister M, Banning A, et al. Flotillin-1/reggie-2 protein plays dual role in activation of receptor-tyrosine kinase/mitogen-activated protein kinase signaling. J Biol Chem. 2012; 287(10): 7265-7278, doi: 10.1074/jbc.M111.287599, indexed in Pubmed: 22232557.

79. Ludwig A, Otto GP, Riento K, et al. Flotillin microdomains interact with the cortical cytoskeleton to control uropod formation and neutrophil recruitment. J Cell Biol. 2010; 191(4): 771-781, doi: 10.1083/jcb.201005140, indexed in Pubmed: 21059848

80. Nakamura H, Sudo T, Tsuiki H, et al. Identification of a novel human homolog of the Drosophila dlg, P-dlg, specifically 
expressed in the gland tissues and interacting with p55. FEBS Lett. 1998; 433(1-2): 63-67, indexed in Pubmed: 9738934.

81. Mburu P, Kikkawa Y, Townsend S, et al. Whirlin complexes with p55 at the stereocilia tip during hair cell development. Proc Natl Acad Sci U S A. 2006; 103(29): 10973-10978, doi: 10.1073/pnas.0600923103, indexed in Pubmed: 16829577.

82. Quinn BJ, Welch EJ, Kim AC, et al. Erythrocyte scaffolding protein p55/MPP1 functions as an essential regulator of neutrophil polarity. Proc Natl Acad Sci U S A. 2009; 106(47): 19842-19847, doi: 10.1073/pnas.0906761106, indexed in $\mathrm{Pu}-$ bmed: 19897731.

83. Evans DG. Neurofibromatosis type 2 (NF2): a clinical and molecular review. Orphanet J Rare Dis. 2009; 4: 16, doi: 10.1186/1750-1172-4-16, indexed in Pubmed: 19545378.

84. Listowski MA, Leluk J, Kraszewski S, et al. Cholesterol Interaction with the MAGUK Protein Family Member, MPP1, via CRAC and CRAC-Like Motifs: An In Silico Docking Analysis. PLoS One. 2015; 10(7): e0133141, doi: 10.1371/journal. pone.0133141, indexed in Pubmed: 26186446.

85. Elderdfi M, Zegarlińska J, Jones W, et al. MPP1 interacts with DOPC/SM/Cholesterol in an artificial membrane system using Langmuir-Blodgett monolayer. Gen Physiol Biophys. 2017; 36(4): 443-454, doi: 10.4149/gpb_2017002, indexed in Pubmed: 28653654.
86. Elderdfi M, Sikorski AF. Interaction of membrane palmitoylated protein-1 with model lipid membranes. Gen Physiol Biophys. 2018; 37(6): 603-617, doi: 10.4149/gpb_2018029, indexed in Pubmed: 30547893.

87. Sharma P, Varma R, Sarasij RC, et al. Nanoscale organization of multiple GPI-anchored proteins in living cell membranes. Cell. 2004; 116(4): 577-589, doi: 10.1016/S00928674(04)00167-9, indexed in Pubmed: 14980224.

88. Mayor S, Rao M. Rafts: scale-dependent, active lipid organization at the cell surface. Traffic. 2004; 5(4): 231-240, doi: 10.1111/j.1600-0854.2004.00172.x, indexed in Pubmed: 15030564.

89. Fujita M, Kinoshita T. Structural remodeling of GPI anchors during biosynthesis and after attachment to proteins. FEBS Lett. 2010; 584(9): 1670-1677, doi: 10.1016/j.febslet.2009.10.079, indexed in Pubmed: 19883648.

90. Eggeling C, Ringemann C, Medda R, et al. Direct observation of the nanoscale dynamics of membrane lipids in a living cell. Nature. 2009; 457(7233): 1159-1162, doi: 10.1038/ nature07596, indexed in Pubmed: 19098897.

91. Navarro-Lérida I, Sánchez-Perales S, Calvo M, et al. A palmitoylation switch mechanism regulates Rac1 function and membrane organization. EMBO J. 2012; 31(3): 534-551, doi: 10.1038/emboj.2011.446, indexed in Pubmed: 22157745.

Submitted: 8 February, 2019 Accepted after reviews: 17 April, 2019 Available as AoP: 16 May, 2019 